\title{
Seroprevalence of Leptospirosis in the Feral Cat Population of St. Kitts
}

\author{
Larry Betance", Andrea Peda, Anne Conan, and Jessica Ribeiro
}

Ross University School of Veterinary Medicine, PO Box 334, Basseterre, St Kitts, West Indies 00234

Received: November 9, 2016 / Accepted: February 24, 2017

\begin{abstract}
Leptospirosis is endemic in most of the Caribbean region, and it is considered to be one the most widespread zoonotic diseases in the world. In cats and dogs, the disease is caused by many different serovars. Cats and dogs have frequent interactions with other animal species including humans, thus they are a potential reservoir for transmission. The objective of this study was to evaluate the seroprevalence of Leptospira sp. in cats in St Kitts. During the periods of February 2015 through December 2015, serum, whole blood and urine were collected from a number of feral cats in Saint Kitts. The standard microscopic agglutination test (MAT) was utilized to determine which feral cats were positive for various serovars: Icterohemorrhagiae, Ballum, Bataviae, Canicola, Grippotyphosa, Ictero, and Pomona. Polymerase chain immunoreactivity (PCR) was performed on urine samples. Out of the 103 feral cats tested, seven cats were MAT positive to one serovar. The overall seroprevalence was estimated at $6.9 \%$ (Confidence Interval: $1.9 \%-11.9 \%$ ). One of the MAT positive cats also tested PCR positive. Although the seroprevalence is low, this study detected an exposure of cats to Leptospira spp. in St Kitts. Our study is the first published seroprevalence survey of Leptospirosis in cats on the Caribbean island of Saint Kitts.
\end{abstract}

Key words: Leptospirosis, cats, Leptospira, St. Kitts, feral, seroprevalence.

* Corresponding author: lbetance@rossvet.edu.kn

\section{Introduction}

Leptospirosis is a zoonotic bacterial disease that affects numerous animal hosts including humans, wildlife and domestic animals (Richardson and Gauthier, 2003). All pathogenic strains occur from the spirochete Leptospira interrogans and over 250 pathogenic serovars have been identified (Adler and de la Pena Moctezuma, 2010). Serovars are maintained in nature by numerous sub-clinically infected wild and domestic animal reservoir hosts that serve as a potential source of infection (Jamshidi et al., 2009).

Transmission of Leptospira between hosts occurs by direct or indirect contact. Direct transmission occurs through contact of infected urine, venereal transfer, bite wounds, or ingestion of infected tissue. Indirect transmission occurs through exposure of animals to contaminated water sources, soil, or food (Sonja et al., 20144). Human Leptospirosis is endemic in most of the Caribbean region, with rodents being the source of most of the infections (Everard and Everard, 1993). In the Caribbean island of St. Kitts, there is limited information of the presence of Leptospira in animals (Everard and Everard, 1993; Loftis et al., 2014; Martinez, 2012).

The Leptospira servovars Canicola, Grippotyphosa, Icterohemorrhagiae, Bataviae, and Pomona have all been isolated in cats (Aedo and Smith, 2014). Cats have been shown to become infected with Leptospira by hunting or coming into contact with the urine of wild or farm animals (Arbour et al., 2012; Larsson et al., 1984). The prevalence of clinical leptospirosis is low in cats, but outdoor cats have a higher seroprevalence than indoor cats (Jamshidi et al., 2009). In comparing rural and ur- 
ban environments, one study showed an increased prevalence of nearly $25 \%$ (Aedo and Smith, 2014). Although cats are less commonly affected with Leptospirosis than dogs, there is no evidence to suggest that they are less capable of spreading the disease (Everard et al., 1979). Epidemiological studies have linked interactions with cats as a risk factor for human Leptospirosis (Lapointe et al., 2013). It is important to determine the prevalence of leptospirosis in feral cats on St. Kitts in order to determine if the incidence of leptospirosis in cat carriers can be linked to leptospirosis in other animals. It is equally important to determine if cats play a role in transmission of disease, and if cats pose a risk of zoonosis. In addition, it would be beneficial to determine if there are any Leptospira serovars on the island of St. Kitts that can be pathogenic specifically to cats. The objective of this study was to conduct a seroprevalence survey of Leptospira in feral cats on the island of Saint Kitts.

\section{Materials and Methods}

Ross University School of Veterinary Medicine (RUSVM) is located on the Caribbean island of St. Kitts. The Feral Cat Project (FCP), run by RUSVM students, is an officially recognized nonprofit organization that strives to increase the welfare of the feral cat population in St. Kitts. A task of this organization is to trap and sterilize feral cats from various locations in St. Kitts. The organization holds approximately four sterilization campaigns (or "Spay days") during a 4-month academic period. The "Spay days" occur on Saturdays in a facility adjacent to the Ross University Veterinary Clinic (RUVC). The FCP team traps feral cats during the week before the Spay day. Traps were placed in various locations where there was a large population of feral cats. The trapped cats are identified by the location they were trapped, and then given an identification number. The cats were then evaluated through observation for overall general health. Once deemed healthy, these cats were anesthetized using a FCP standardized protocol which includes an injectable premedication, endotracheal tube intubation, and Isoflurane gas maintenance.

Following RUSVM standard blood collection protocol, cats had one side of their neck shaved and aseptically prepped with alcohol. Using a 22-gauge needle and $6 \mathrm{~mL}$ syringe, a maximum of $4 \mathrm{~mL}$ of blood was collected from their jugular vein. If blood could not be obtained from the jugular vein, the medial saphenous vein was utilized for collection. After collection, the blood was immediately transferred into a $2.7 \mathrm{~mL}$ plain tube (red top) and a $2.7 \mathrm{~mL}$ EDTA tube with appropriate identification numbers and date. A few drops of blood were retained in the syringe and utilized for testing of FELV/FIV with an Idexx Laboratoriesa snap test. The caudal abdomen was palpated to locate the bladder and a cystocentesis was also performed. Approximately one to five $\mathrm{ml}$ of urine was collected using a similar 22-gauge needle and bcc syringe. The collected urine was then placed in a $2.7 \mathrm{~mL}$ plain tube.

Complete blood counts $(C B C)$ with manual differential counts were performed on the samples the same day of collection. All samples were stored after testing in a refrigerator kept at $5{ }^{\circ} \mathrm{C}$. A Vet Scan HM5 or Vet Scan HM5 touch was utilized to run the complete blood counts. A blood smear was made and evaluated microscopically to perform a manual differential count on each sample.

Polymerase chain reaction (PCR) testing was done on every cat in the study. A majority of the PCR tests were performed on urine samples. Four PCR tests were performed on EDTA (Ethylenediaminetetraacetic acid) samples due to the inability to collect urine samples from these patients. Samples were centrifuged the same day and stored at $4^{\circ} \mathrm{C}$.

Volumes of urine (whole blood EDTA were used in cases where no urine was received) samples were centrifuged at 20,000xg (gravitation force) for 5 minutes at $25^{\circ} \mathrm{C}$. The supernatant was discarded and the pellet was washed twice with $500 \mu$ l sterile $1 \mathrm{X}$ phosphate-buffered saline (PBS). Each sample was centrifuged between each wash. The pellet was re-suspended in 200 $\mu \mathrm{I}$ PBS and then the extraction of the DNA was performed. DNA was extracted using the DNeasy Blood and Tissue kitb following the manufacturer's directions for protocol "Purification of Total DNA from Animal Blood or Cells (Spin Column)"c. "Spin Column" is a protocol designed for purification of total DNA from animal blood and urine. The samples were kept at $-20^{\circ} \mathrm{C}$ prior to $\mathrm{PCR}$ analysis.

Serum samples were placed into red top tubes and were stored at $5{ }^{\circ} \mathrm{C}$ until microscopic agglutination test (MAT) was able to be performed. The testing was performed under routine procedure. The serum was diluted by taking $20 \mu \mathrm{L}$ of serum and adding $980 \mu \mathrm{L}$ of PBS. The sample was then mixed thoroughly and then $50 \mu \mathrm{L}$ of each serum sample were inoculated into each well of a 96-well plate. When the number of serum samples were not adequate to fill the entire 92 wells of the plate, PBS was added to the remaining wells to act as the negative control. Each entire plate was then inoculated with $50 \mu \mathrm{L}$ of one serovar. Plates were incubated at $29{ }^{\circ} \mathrm{C}$ for $1.5-2 \mathrm{hrs}$. Each serum sample was tested with each of the six serovars. After incubation, dark field microscopy was used to observe for agglutination. $50 \%$ agglutination was given a value of $2+$ and considered positive for a 1:100 titer. Anything lower than 50\% (or less than $2+$ ) was considered negative. Dilutions above 1:100 were not performed for this study as it meant to serve as an exposure prevalence study and not a study looking for active infection in feral cats.

Data analyses was performed using $R$ softwared. Fisher's exact test was used to compare seroprevalence by gender and age category. $C B C$ results were compared between seropositive and seronegative cats using a Wilcoxon rank test. The map was done using ArcGIS v10.3.1.

\section{Results}

During the time period of February 28, 2015 through December 5, 2015, 103 cats were trapped and collected from twenty-one different locations in St. Kitts. MAT could not be performed on one female adult. This individual was excluded from the analyses. With 7 MAT positive cats, the seroprevalence of Leptospirosis was of $6.9 \%$ (Confidence Interval Cl: $1.9 \%$ $11.9 \%$ ) (Table 1). MAT positive cats were found in five locations (Figure 1). Fifty three cats $(53 \%)$ were male and $50(47 \%)$ 
Table 1. Demographic and laboratory characteristics of MAT positive cats.

\begin{tabular}{lllllll}
\hline Site of Trapping & Location & Gender & Age & PCR & Serovar & $\begin{array}{l}\text { Total Number of Cats } \\
\text { Trapped at this Site }\end{array}$ \\
\hline Lime Kiln & Boyds/West Farm & $F$ & $<1$ year & Positive & Bataviae & 10 \\
New Road & New Road & $F$ & 12 weeks & Negative & Bataviae & 4 \\
West Farm & Boyds/West Farm & $M$ & $>1$ year & Negative & Pomona & 11 \\
Half Moon Bay & Half Moon Bay & $M$ & $<1$ year & Negative & Bataviae & 4 \\
Marriott Hotel & Frigate Bay & F & $>1$ year & Negative & Pomona & 5 \\
Camps & Camps/Fortland & $M$ & 1 year & Negative & Pomona & 5 \\
Frigate Bay & Frigate Bay & F & 1 year & Negative & Ballum & 5 \\
\hline
\end{tabular}

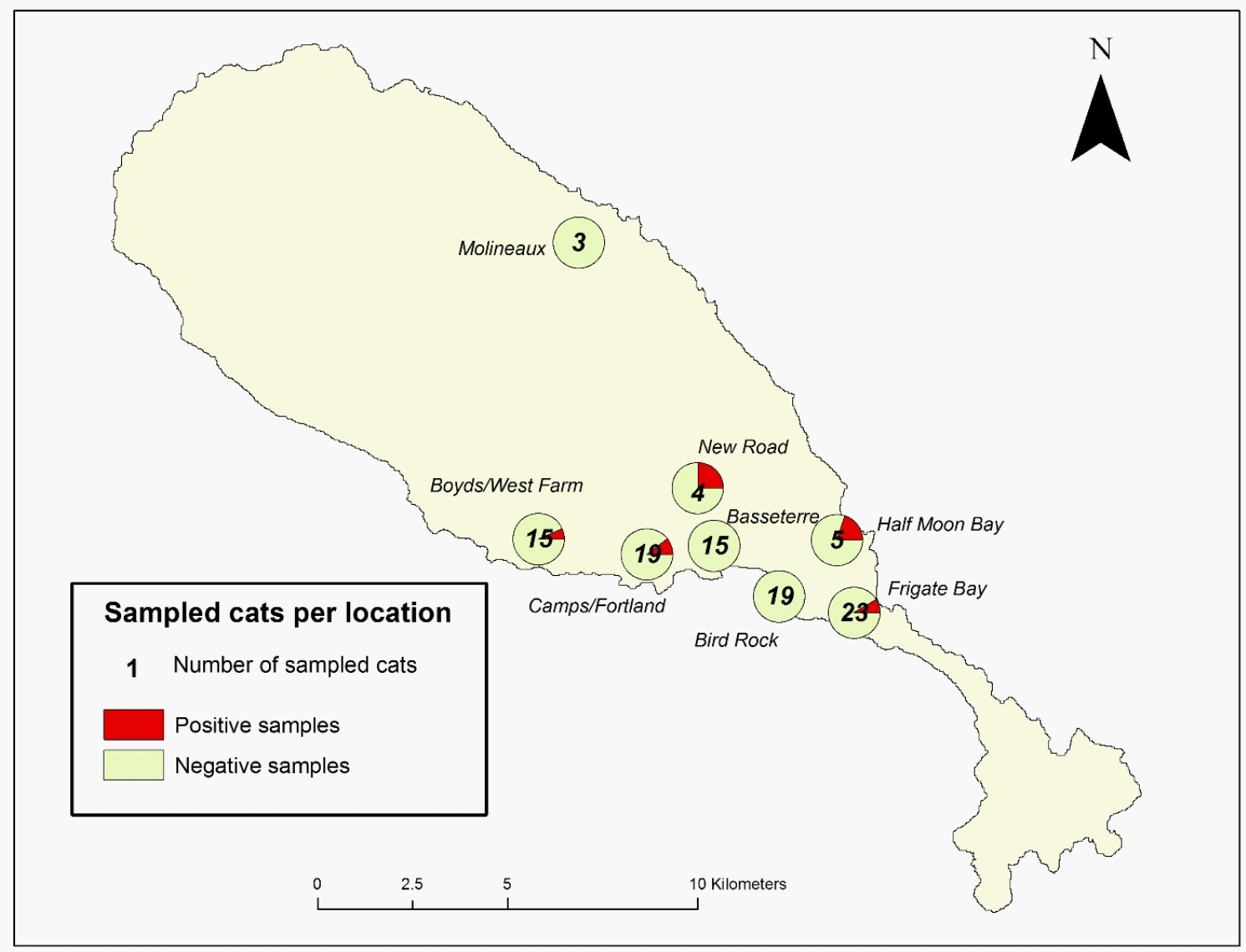

Figure 1. Location of trapping of FCP cats and MAT positive cats in St Kitts.

were female. Cats' ages were estimated, aged 3 months to 3 years old. Fifty one $(50 \%)$ were one year old and more. There was no difference of prevalence between male and female cats $(5.6 \%$ vs. $8.3 \%, p=0.7)$ or between less than one year old and greater than one year old cats $(p=1)$. Detected serovars were Pomona (4 times), Bataviae (3 times), and Ballum (1 time) (Table 1). Of the 7 MAT positive cats, one PCR was positive: a young $(<1$ year old) female positive seropositive to Bataviae. Complete blood counts were performed on the six of the MAT positive cats and 84 of the MAT negative cats. The MAT positive cats in our study did not have appreciable $C B C$ changes aside from a mild eosinophilia in five of them $(p=0.3)$. Significantly lower platelet count $(p=0.005)$ and higher monocyte count $(p=0.03)$ were detected in MAT negative cats.

\section{Discussion}

Our study is the first to estimate the seroprevalence of Leptospirosis in cats in St Kitts. The overall seroprevalence was of 6.9 $\%$ (Confidence Interval Cl: $1.9 \%-11.9 \%$ ). This result is coherent with what Azocar-Aedo et al. found in domestic cats in Chile (8.1\% for apparent seroprevalence) (Aedo and Smith, 2014). Serosurveys typically show $<10 \%$ feline exposure rates with outdoor cats having highest probability of exposure (Greene, 2012). The seroprevalence found in St. Kitts is equivalent.

There are very few studies on the prevalence of leptospirosis in cats and from what is available there is only one study found in the literature on the prevalence of feline leptospirosis in the Caribbean on the island of Trinidad. This study investigated 
both cat and dog populations. There were limited numbers of cats involved in the study, but the study concluded that cats are less commonly infected than dogs (Everard et al., 1979). There is no clear data on whether or not felines are significant carriers in spreading the disease to other animals or humans. The role of cats in the epidemiology of Leptospirosis has not been investigated. Leptospirosis is much better described in dogs since clinical disease in cats is usually mild or inapparent.

Of the 7 positive results (2+ agglutination or greater which is equivalent to a titer of at least 1:100), 3 were male and 4 were female. Initially it was hypothesized that males would be more prevalent to Leptospirosis compared to females because they are more likely to assert dominance by fighting with other cats and tend to hunt aggressively, increasing their exposure to Leptospira (Larsson et al., 1984). Yet, the results did not show a statistical difference in distribution of males and females in exposure to Leptospira.

In our study, all cats, that tested MAT positive, came from different colonies around the island, so no single neighborhood was over-represented. There was only 1 PCR positive cat, thus no conclusions can be drawn by location. All cats tested were either feral kittens or young adults. No geriatric felines were tested so we cannot draw conclusions based on age, however it is known that this is more commonly a disease of adult cats (Arbour et al., 2012; Larsson et al., 1984).

A total of $7 \%$ of all cats tested were seropositive at a titer of $1: 100$ indicating clinically insignificant disease. Usually a titer of 1:200 or greater is considered an active infection (Lapointe et al., 2013). Therefore, it appears this population of cats would pose a low risk to zoonotic spread of disease; however, this remains a possibility. We cannot determine if this translates to client owned indoor/outdoor cats since they were not included in the study. The serovars found in this study including Pomona and Ballum have been found to cause disease in other animals as well as people.During this study, three serovars were detected: Pomona, Ballum and Bataviae. Leptospira are classified into 24 serogroups and over 200 serovars according to their different lipopolysaccharides (Chirathaworn et al., 2014). Serovars from serogroups Canicola, Grippotyphosa and Pomona have been isolated from cats (Greene, 2012 ). Serogroups Autumnalis, Bratislava, Canicola, Grippotyphosa, Hardjo, Icterohemorrhagiae and Pomona have all been found in feral and shelter cats (Greene, 2012).

A young female seropositive to Bataviae was positive to the PCR. PCR can determine pathologic from non-pathologic organisms, but it cannot differentiate which serovar is present in a patient (Greene, 2012). While this test is very sensitive, false negatives may be obtained due to timing of testing or recent antibiotic use. False positive results are also possible therefore clinical signs should always be considered. In this study, we were unable to do this since the cats were feral and no history or follow up were conducted. Besides, the positive cat detected in our study had no $C B C$ changes other than an eosinophilia, which may have been associated with endoparasitism, ectoparasitism, or other hypersensitivity reactions. Cats are normally incidental hosts to serovar Bataviae.

MAT cannot differentiate between organisms at a serovar level, but it is serogroup specific. A positive test cannot differentiate between active infection, carrier status, or previous exposure. It also does not differentiate between recently vaccinated patients (Villanueva et al., 2010). Serovar cross reactivity is a significant problem because it makes it very difficult to confirm which serovars are present. The serovars can cross react on MAT testing naturally when they are closely related. In addition, cross reactivity can occur if there is more than one serovar present in the host, and cross reactivity can also occur in a host who was previously infected with one serovar then recently exposed to another serovar. This alone prevents us from confirming definitive outcome assessments in this study. In order to differentiate from cross reactivity, recommendations are to perform DNA based or molecular typing. This can help differentiate closely related serovars and confirm the presence of specific serovars (Villanueva et al., 2010).

\section{Limitations to this Study}

Feral cats were tested from 21 sites in 8 locations in St. Kitts, but there is still a large geographical area of St. Kitts that was not represented in this study, which could skew the results. Cats were trapped from selected areas with known high feral cat populations. There may be other areas of St. Kitts where the cat populations are not high, but could have a higher or lower incidence of exposure to Leptospirosis. Ideally, trapping cats from all neighborhoods of the island may provide a better representation of the incidence of exposure in the entire feral cat population as a whole.

There was unknown presence of clinical illness in these feral cats due to lack of history or long term observation. It is unknown if there were any renal or hepatic changes in positive cats as no serum chemistries were performed. As mentioned earlier, we did not have a history of the cats trapped for this study. Factors including whether they are friendly or truly feral, there living habitat, and previous medical history, could all help in determining clinical illness verses exposure.

\section{Conclusion}

This is the first study done on the island of Saint Kitts to determine the prevalence of exposure to Leptospirosis in the feral cat population. The seroprevalence was low $(<10 \%)$. Even though seven of the positive MAT results had a 2+ antibody titer, these results could not be validated as true positives due to the inability to obtain a history and follow up on these feral cats. Future studies could include complete histories and post testing follow-ups, which would help to substantiate positive and negative titers. Future studies should be done in order to estimate the prevalence of this disease on the island of Saint Kitts and clarify the role cats play in transmission of leptospirosis to other animals and/or humans.

\section{Notes}

a: Idexx Laboratories, Inc. One IDEXX Drive Westbrook, Maine, 


\section{2}

b: DNeasy Blood and Tissue Handbook, July 2006, www.qiagen.com.

c: ThermoFisher Scientific: Applied Biosystems Real-Time PCR Instruments. http://www3.appliedbiosystems.com/cms/groups/ mcb_support/documents/generaldocuments/cms_041280.pdf $\mathrm{d}$ : $\mathrm{R}$ core team. A language and environment for statistical computing. R Foundation for Statistical Computing, Vienna, Austria 2014. Available from: http://www.R-project.org.

\section{Acknowledgements}

The authors would like to acknowledge and give special thanks to Trellor Fraites (Director of Laboratory Services) and Julienne Rawlins (Senior Laboratory Service Technician) for all their hard work and dedication in providing critical laboratory data essential for this project.

\section{References}

Adler B and A de la Peña Moctezuma (2010). Leptospira and leptospirosis. Veterinary microbiology 140 (3): 287-296.

Aedo LA and HL Smits (2014) Leptospirosis in dogs and cats: epidemiology, clinical disease, zoonotic implications and prevention. Archivos de Medicina Veterinaria 46 (3): 337-348.

Arbour J, MC Blais, L Carioto, and D Sylvestre (2012) Clinical leptospirosis in three cats (2001-2009). Journal of the American Animal Hospital Association 48 (4): 256-260.

Chirathaworn C, R Inwattana, Y Poovorawan, and D Suwancharoen (2014) Interpretation of microscopic agglutination test for leptospirosis diagnosis and seroprevalence. Asian Pacific Journal of Tropical Biomedicine 4: S162-S164.
Everard CO, EP Cazabon, DW Dreesen, and CR Sulzer (1979) Leptospirosis in dogs and cats on the Island of Trinidad: West Indies. International Journal of Zoonoses 6 (1): 33-40.

Everard JD and COR Everard (1993) Leptospirosis in the Caribbean. Reviews in Medical Microbiology 4 (2): 114.

Greene CE (2012) Leptospirosis. In Infectious diseases of the dog and cat (4th ed., pp. 431-447). St. Louis, MO: Elsevier/Saunders.

Jamshidi S, MA Akhavizadegan, N Maazi, AG Ali, and S Bokaie (2009) Serologic study of feline leptospirosis in Tehran, Iran. Iranian Journal of Microbiology 1 (2): 32-36.

Lapointe C, I Plamondon, and M Dunn (2013) Feline leptospirosis serosurvey from a Quebec referral hospital. The Canadian Veterinary Journal 54 (5): 497.

Larsson CE, CA Santa Rosa, MK Hagiwara, GV Paim, and JL Guerra (1984) Prevalence of feline leptospirosis: serologic survey and attempts of isolation and demonstration of the agent. International Journal of Zoonoses 11 (2): 161-169.

Loftis A, F Castillo-Alcala, L Bogdanovic, and A Verma (2014) Fatal Canine Leptospirosis on St. Kitts. Veterinary Sciences 1 (3): 150158.

Martinez (2012) Saint Kitts and Nevis. In Health in the Americas: Regional Overview and Country Profiles (2012 ed., Vol. Country, pp. 548-561). Scientific and Technical Publication.

Richardson DJ and JL Gauthier (2003) A serosurvey of leptospirosis in Connecticut peridomestic wildlife. Vector-Borne and Zoonotic Diseases 3 (4): 187-193.

Sonja O, R Sonja, S Nataša, B Danica, V Slobodanka, and V Miroslav (2014) Seroprevalence of cat leptospirosis in Belgrade (Serbia). Acta Veterinaria 64 (4): 510-518.

Villanueva SY, H Ezoe, RA Baterna, Y Yanagihara, M Muto, N Koizumi, and NG Gloriani (2010) Serologic and molecular studies of Leptospira and leptospirosis among rats in the Philippines. The American Journal of Tropical Medicine and Hygiene 82 (5): 889-898. 